\title{
Politique
}

\section{Crise de l'État ou crise du capitalisme}

\section{Bernard Élie}

Numéro 3, hiver 1983

La crise des finances publiques au Québec

URI : https://id.erudit.org/iderudit/040420ar

DOI : https://doi.org/10.7202/040420ar

Aller au sommaire du numéro

Éditeur(s)

Société québécoise de science politique

ISSN

0711-608X (imprimé)

1918-6584 (numérique)

Découvrir la revue

Citer cet article

Élie, B. (1983). Crise de l'État ou crise du capitalisme. Politique, (3), 117-127.

https://doi.org/10.7202/040420ar d'utilisation que vous pouvez consulter en ligne.

https://apropos.erudit.org/fr/usagers/politique-dutilisation/ 


\title{
Crise de l'État ou crise du capitalisme
}

\author{
Bernard Élie \\ Université du Québec à Montréal
}

L'État serait en crise financière, voilà un diagnostic grave, sommes-nous en faillite? Cette analyse est faite par les économistes libéraux et les organisations patronales; le remède c'est l'austérité; "rationalisation de la gestion et réduction des dépenses de l'État", comme s'il s'agissait d'une entreprise comme les autres, l'État doit faire une pause. Rester à ce premier niveau d'analyse comptable, c'est s'empêcher de voir le pourquoi de cette prescription faite par les médecins économistes ${ }^{1}$ et surtout d'envisager de nouvelles pistes de sortie de crise. Si le rôle de l'État est ainsi remis en cause, c'est peut-être que le «contrat social» mis sur pied depuis la guerre et dont l'État était le garant, constitue maintenant une entrave pour le capital, lui-même en crise.

La crise financière de l'État n'est pas uniquement une opération technique que quelques ajustements permettraient de résoudre. Les difficultés du gouvernement du Québec et l'énorme déficit du fédéral ne sont pas seulement des «hiatus» temporaires que de saines mesures d'austérité feront disparaître. Ce qui est remarquable ici, ce n'est pas tant l'ampleur des

1. Milton Friedman est particulièrement friand des parallèles entre la médecine et l'économie. 
«trous» que l'horreur qu'ils suscitent brusquement. Horreur qui permet d'identifier un coupable en dégageant son irresponsabilité, mais surtout de faire abstraction du vrai problème. Nous n'avons pas l'intention ici de faire une guerre de chiffres ou d'arpentage de trous, mais plutôt de montrer comment sous prétexte d'une crise financière de l'État, le patronnat tente d'opérer une vaste redistribution des revenus en sa faveur et de se faire confirmer dans son action par les gouvernements. Ce qui n'a rien d'immoral dans une économie capitaliste décentralisée où les décisions doivent émaner d'un petit nombre. Rappelons que les tentatives actuelles d'augmentation de «productivité » ont commencé d'abord dans le secteur privé et qu'elles ont conduit à l'augmentation de l'insécurité des travailleurs. Cette "précarisation» du travail se manifeste par la réduction des salaires, la réduction du temps de travail, le travail à temps partagé, le travail à domicile, les mises à pied temporaires ou permanentes, la multiplication des emplois de courte durée et dans tous les cas par la réduction ou l'absence des avantages dits sociaux.

\section{La nécessité d'une nouvelle donne}

Dans le quart de siècle qui suivit la deuxième guerre mondiale l'économie des pays occidentaux a connu une croissance et un développement sans précédent dans son histoire, on en a même oublié les crises. La base de ce développement était les États-Unis, son déploiement a été assuré par l'expansion des firmes multinationales, la reconstruction des économies européennes et japonaises, la mainmise sur les richesses du tiersmonde et la consommation de masse. Les premiers toussottements au début des années soixante se manifestèrent par un blocage de la productivité et une baisse des profits ${ }^{2}$. Pendant

2. AGLIETTA, Michel, «Les nouvelles perspectives du capitalisme américain » Économie et statistique, No. 77, avril 1976. 
toute cette période les gouvernements ont eu comme rôle d'assurer la stabilité sociale indispensable à cette croissance. L'ensemble des politiques de revenu, de stabilisation, élaboré dès 1934 avec le New Deal de Roosevelt, a eu pour conséquence de démocratiser le capitalisme en lui assurant une base de consommation plus large. L'action de l'État a pris deux formes: une gestion de la force de travail et une gestion de la monnaie ${ }^{3}$.

La gestion de la force de travail a pris la forme d'une série de mesures qui devraient permettre la reproduction de la force de travail même hors du marché du travail, l'adaptation de cette force de travail aux besoins de la production et un niveau de demande minimal (assurance-chômage, pension de vieillesse, normes minimales de travail, reconnaissance des syndicats). L'État prenait une part active dans la redistribution des revenus et assurait ainsi un rôle d'harmonisation sociale. L'État devenait un partenaire attentif et utile aux besoins du capital. La gestion de la monnaie devrait homogénéiser l'ensemble des transactions privées et là encore assurer la stabilité de la circulation de la production. Sur le plan national le rôle de prêteur de dernier recours donné aux banques centrales, l'instauration de l'assurance dépôt, le contrôle de la gestion des banques privées permettrait une certaine régulation, surtout dans un système monétaire à cours forcé. Les faillites bancaires et les désordres monétaires des années 20 et 30 seraient empêchés en reportant la contrainte monétaire sur l'État. Sur le plan international la nouvelle règlementation établie à Bretton Woods, sous la tutelle des États-Unis, créait un cadre plus propice à la circulation des marchandises et des capitaux.

Tout semblait être en place pour la disparition des crises, ou tout au moins, l'atténuation des cycles économiques. L'État devenait le grand régulateur.

3. BRUNHOFF, Suzanne de, État et Capital, Paris PUG/Maspero, 1976. 
«L'élargissement des fonctions de l'État, qu'implique la responsabilité d'ajuster l'une à l'autre la propension à consommer et l'incitation à investir, semblerait à un publiciste du XIX $\mathrm{X}^{\mathrm{e}}$ siècle ou à un financier américain d'aujourd'hui une horrible infraction aux principes individualistes. Cet élargissement nous apparaît au contraire et comme le seul moyen possible d'éviter une complète destruction des institutions économiques actuelles et comme la condition d'un fructueux exercice de l'initiative individuelle» ${ }^{4}$.

Aujourd'hui, ce mode de régulation apparaît comme des concessions inacceptables à la classe ouvrière dont une partie a ainsi accédé à des conditions d'existence et à des niveaux de vie jusque là réservés à une minorité. Ces acquis, signes de la transformation des rapports sociaux qui ont donné naissance à «L'ÉtatProvidence», sont tout à fait contraires aux fondements d'une économie libérale basée sur la libre entreprise. Dès que la crise est revenue, le capital a réclamé une nouvelle donne. Les critiques de plus en plus fortes des économistes libéraux ont été plus qu'entendues, elles ont été endossées par les gouvernements, même les plus «sociaux-démocrates». En centrant leurs attaques contre l'État, ce sont les bases mêmes de l'ancien contrat social qui sont remises en cause. Il ne s'agit pas d'abolir l'État ${ }^{5}$, bien au contraire, l'État doit continuer à maintenir l'ordre social et s'occuper des fous ${ }^{6}$, mais l'État ne doit plus limiter l'action du secteur privé. La fin de l'entracte keynésien est sonnée, les politiques d'austérité sont de retour.

\section{L'austérité de nos gouvernements}

La crise capitaliste est l'occasion d'une vaste redistribution des revenus dans notre société. Les États canadien et québécois

4. KEYNES, J.M. Théorie générale de l'emploi, de l'intérêt et de la monnaie, Paris, Petite coll. Payot, 1971, 373.

5. LEPAGE, Henri, Demain le capitalisme, Paris, le Livre de Poche, 1978.

6. FRIEDMAN, Milton, cité par WELDON, Jack, «Une critique de la privatisation" in La crise économique et sa gestion, Acte du colloque de l'A.E.P., Montréal, Boréal Express, 1982, 204. 
participent à ce processus par leurs politiques d'austérité. La stratégie utilisée est la culpabilisation collective et la division des travailleurs(euses), les non-syndiqué(e)s contre les syndiqué(e)s, les travailleurs(euses) du secteur privé contre ceux(celles) du secteur public, les travailleurs(euses) précarisé(e)s contre ceux(celles) qui ont un emploi «normal », les femmes contre les hommes, les jeunes contre les plus âgés...

L'austérité est devenue la doctrine économique officielle de nos gouvernements. Cette doctrine, qui conduit à la réduction du pouvoir d'achat des salariés et à des coupures dans les programmes sociaux, nous suggère que l'ensemble de nos problèmes actuels est dû essentiellement à un dérèglement social, donc a-économique, ou à des causes externes (prix du pétrole, climat, politique monétaire US ou canadienne). En un mot, nous avons été amenés, en particulier nous les Québécois, à vivre au-dessus de nos moyens et nous devons en subir les conséquences. Dans une telle logique, la crise économique, ou la récession pour les optimistes, ne peut venir du secteur de la production, du secteur réel, parce que «l'économie québécoise est une économie fondamentalement saine ${ }^{7}$. Essentiellement, une telle vision est un acte de foi dans l'économie de marché, de libre entreprise, et un retour à l'orthodoxie économique prékeynésienne. Abandonner le rôle que l'État s'était donné, suivre les « lois» du marché, voilà la solution «nouvelle» pour revenir à un monde meilleur. Concrètement, l'austérité signifie que l'État doit perdre son rôle de définisseur des normes sociales de conditions de vie et laisser le secteur privé fixer les revenus des salariés en fonction de ses propres priorités. L'alignement des revenus des employés du secteur public sur ceux du secteur

7. Secrétariat permanent des conférences socio-économiques, "L'état de la situation socio-économique», "La Conférence au sommet», Québec 1982, tenue les 5-6-7 avril 1982, 3. 
privé et la réduction, relative, du déficit de l'État ne sont que l'expression de cette orientation qui conduit à une redistribution de la richesse nationale en faveur des entreprises. Mais toutes les entreprises ne font pas l'objet du même traitement, celles de hautes technologies, exportatrices ou du secteur énergétique, ont un traitement de faveur. Notons en passant que lorsque les comparaisons salariales entre les secteurs privé et public sont possibles, ce sont les femmes syndiquées du secteur public et les hommes syndiqués du secteur privé qui ont la meilleure position $^{8}$. L'alignement des salaires doit donc se faire, pour nos gouvernements, en fonction des emplois non-syndiqués et les plus précaires du secteur privé.

Cette vaste opération de redistribution du revenu va s'accentuer au fur et à mesure que la crise économique s'approfondira. Le pouvoir d'achat du salarié moyen au Canada, déjà, a baissé de $11 \%$ depuis 5 ans $^{9}$ et on nous annonce au Québec une baisse de près de $19 \%$ des salaires des employés de l'État. On nous présente aussi la réduction «volontaire» des salaires, ou encore, le travail partagé comme étant le moyen de sauver l'emploi. Tout ceci a-t-il pour but de nous faire croire que la baisse du niveau de salaire réel pourrait permettre l'augmentation de l'emploi? Les États du Québec et du Canada, ici, n'innovent pas. Déjà le secteur privé a «convaincu» une partie de ses travailleurs de réduire leur salaire ou leur temps de travail pour sauver des emplois! L'État ne fait que s'aligner. L'ensemble de ces mesures vise à rétablir la rentabilité des entreprises qui se voient étrangler par les hauts taux d'intérêt et la chute de leurs ventes. Cette vision à courte vue, qui consiste à hausser les profits au détriment des salaires, amènera une situation dans

8. BEAUCAGE, André, ÉLIE, Bernard, «Les comparaisons de la rémunération entre les secteurs public et privé », Le Devoir, Montréal, 10 avril 1979.

9. INGERMAN, S., «La crise et les politiques économiques au Canada", in La crise économique et sa gestion, A.E.P. Boréal Express, 44. 
l'emploi plus catastrophique et de plus grandes difficultés pour les entreprises, surtout les plus faibles. Certes, on nous vante les vertus de l'exportation pour suppléer à l'insuffisance de la demande nationale, ou les investissements dans les secteurs de «pointe» peu créateurs d'emploi, mais ces stratégies ne pourront que se heurter aux mêmes blocages de la demande, ici mondiale, et à la concurrence internationale de plus en plus dure.

La vision économique que l'on nous propose, en plus de manquer d'imagination et de rester dans des schémas dépassés, fait abstraction de la nécessité d'une restructuration en profondeur de notre société et impose à la société québécoise un coût social énorme et peut-être inutile. Ne mentionnons que la situation des jeunes, avec un taux de chômage de $24.0 \%$ en juin 82 pour les moins de 24 ans ${ }^{10}$, à qui l'on interdit l'accès à la dignité par un travail valorisé et créateur. Le blocage qui est fait à cette génération est une hypothèque beaucoup plus grave qu'un déficit de 3 milliards \$. Le gouvernement du Québec a des difficultés financières qui vont s'accentuer avec la baisse de l'emploi et des revenus, mais le caractère obsessionnel que prend le fameux «trou» lui fait perdre toute capacité d'envisager une politique économique, pas nécessairement sortie de crise, mais tout au moins de résistance plus «sociale» à la crise.

\section{Les solutions}

L'insécurité des travailleurs(euses) constitue un des fondements du système capitaliste, le remettre en cause, c'est nier le droit des propriétaires des moyens de production de disposer librement de leur main-d'œuvre, c'est contester le droit de propriété. L'offensive actuelle contre les travailleurs(euses) - au 
travail ou non - s'inscrit dans l'application de ce droit. Les stratégies utilisées actuellement dont la base théorique est le monétarisme, ont comme objectif d'éliminer les obstacles à cette insécurité, l'État ( Providence») est ici une cible de choix. Ces attaques devront conduire à un partage de la valeur sociale ajoutée en faveur du capital. On nous présente même l'État comme la cause de la crise, par ses règlementations «antimarché» ou par son déficit.

Face aux coûts économiques et sociaux du chômage ${ }^{11}$ et à la brutalité des moyens proposés par les monétaristes, les néokeynésiens envisagent des solutions plus douces à la nouvelle répartition des revenus. Eux aussi ne remettent pas en cause l'insécurité inhérente au marché du travail. Ils ${ }^{12}$ nous suggèrent une stratégie plus ordonnée pour revenir à l'équilibre d'aprèsguerre qui n'aurait été possible, selon eux, que grâce à l'action régulatrice de l'État, implicite au modèle de Keynes. Essentiellement, leurs propositions d'ordre mécanique sont les suivantes: l'établissement d'un contrôle des prix et des salaires, pour une durée de trois ans, l'introduction de légers stimulants fiscaux et la baisse des taux d'intérêts. Cette dernière mesure, en plus d'alléger les charges des entreprises, entraînerait une chute de notre taux de change face au dollar américain, et ainsi, une hausse de nos exportations et de l'emploi au Canada. Donc, exportons notre chômage. La première mesure, les contrôles, permettrait de juguler l'inflation puisqu'elle est essentiellement importée pour les néo-keynésiens. Dans ce type de mesures, seuls les salaires sont véritablement contrôlables, ou contrôlés, la détermination des prix est soit «extérieure» ou trop «complexe» pour être gérée. Le contrôle des salaires est l'élément

11. Voir à ce sujet les études de Diane Bellemare et de Lise Poulin-Simon.

12. BARBER, Clarence L. et McCALLUM, John C.P., Unemployment and Inflation - The Canadian Experience, Ottawa, Institut canadien de politique économique, 1980. 
ordonné de l'intervention de l'État dans la redistribution des revenus, la rémunération des salariés seule perd du terrain face à l'inflation. En ce qui concerne les stimulants fiscaux, ils semblent être là pour respecter l'héritage keynésien, leur légèreté montre bien comment les critiques contre l'État-providence ont bien été intégrées là aussi.

Dans le cadre de l'internationalisation de la crise, il est très difficile, pour ne pas dire impossible, pour un seul pays de résoudre totalement ses difficultés. Ce que nous devons envisager c'est un front de résistance à cette crise au bénéfice de la majorité. Une telle orientation doit passer par une véritable politique de l'emploi, et non plus de main-d'œuvre, axée sur la protection des emplois actuels et sur la création de nouveaux emplois. Que le maintien de l'emploi soit une priorité absolue aussi bien dans le secteur privé que public selon un critère d'efficacité sociale et non pas d'efficacité financière. Que de vastes programmes de rénovations urbaines et de constructions d'équipement collectifs soient entrepris. Mais surtout que la situation des travailleurs(euses) les plus en insécurité soit améliorée, tant au niveau de la stabilité qu'au niveau du revenu. Nous pensons ici aux emplois à temps partiel, aux pigistes, aux travaux à domicile... Que la syndicalisation soit encouragée et valorisée.

Certes, une telle politique ne pourrait qu'augmenter les besoins financiers des gouvernements. Mais ici, en plus d'assurer à l'État un financement plus large, il faut se donner les moyens de contourner les contraintes du marché financier actuel. En ce qui concerne l'État, deux types d'action devraient être entrepris: au niveau fiscal, une augmentation de la taxation sur les hauts revenus et la fermeture des échappatoires fiscaux; au niveau budgétaire, une redistribution des dépenses vers la création d'emploi (réduction des dépenses militaires et des subventions aux grandes entreprises, surtout dans le domaine 
énergétique). Mais avant tout, notre action collective doit se porter sur le marché financier.

Les entreprises canadiennes et québécoises font face à des difficultés de financement énormes alors que jamais l'épargne des Canadiens n'a été aussi élevée. Les intermédiaires financiers jouent un rôle déformant en resserrant leurs conditions de prêts aux entreprises. Ces dernières ont trois sources de financement. L'auto-financement provenant des profits non-distribués et des provisions pour amortissement a chuté de façon importante depuis le début de 1981 (de près de $18 \%$ du P.N.B. à un peu plus de $12 \%$ au premier trimestre 1982) ${ }^{13}$. Le recours au marché des valeurs mobilières a lui aussi été réduit fortement il est devenu peu alléchant, compte tenu des hauts taux d'intérêt. Les emprunts bancaires constituent maintenant la principale source de financement. Ce financement externe représente une charge accrue pour les entreprises, réduisant du même fait leurs capacités propres de financement (les paiements d'intérêt ont atteint 2.3 milliards de $\$$ au ler trimestre de 1980, 2.9 milliards au ler trimestre 1981, et 4.07 milliards au ler trimestre 1982) ${ }^{14}$. D'autre part, ce crédit est en grande partie à court terme rendant la trésorerie des entreprises plus aléatoire.

L'État et les entreprises font donc face à de sérieuses difficultés de liquidité, mais aussi à des conditions de financement très dures. Par contre, l'épargne personnelle des Canadiens touche à de nouveaux sommets (encore plus pour les Québécois). Cette épargne qui se retrouve essentiellement dans les banques à charte et les caisses populaires est prêtée à court terme et à des conditions défavorables pour les emprunteurs. Les structures financières doivent être modifiées pour répondre aux besoins d'une politique de l'emploi.

13. Statistique Canada, Catalogue No. 61-006, ler trimestre 82.

14. Statistique Canada, Catalogue No. 61-003, ler trimestre 82 tableau 4. 
La création d'un fonds national de l'épargne, utilisant une partie de notre épargne et qui accorderait des conditions plus souples aux emprunteurs, est une solution pour réduire les effets catastrophiques sur l'emploi du marché financier actuel. Les souscriptions à ce fonds, garanties par l'État, peuvent prendre plusieurs formes: dépôts à terme, achats d'obligations, elles seront volontaires ou obligatoires et l'intérêt versé aux déposants, sera celui du marché. Cette nationalisation de l'épargne, même partielle, pour satisfaire les besoins collectifs, implique bien sûr un changement, il ne s'agit que d'étendre le rôle de la Caisse de dépôts et de placements du Québec. Décider la création d'un tel fonds indiquerait nettement la volonté de nos gouvernements de ne pas laisser au secteur privé, en particulier financier, la gestion de la crise. 